\title{
Does combined strength training and local vibration improve isometric maximum force? A pilot study
}

\author{
Ruben Goebel ${ }^{1}$ \\ Monoem Haddad ${ }^{1}$ \\ Heinz Kleinöder ${ }^{2}$ \\ Zengyuan Yue $^{3}$ \\ Thomas Heinen ${ }^{4}$ \\ Joachim Mester ${ }^{5}$
}

1 Sport Science Program. College of Arts and Sciences, Qatar University, Doha, Qatar

2 Institute of Training Science and Sport Informatics, German Sport University Cologne, Cologne, Germany

3 The German Research Center, Center of Elite Sport, German Sport University Cologne, Cologne, Germany

4 Faculty of Sport Science, Leipzig University, Leipzig, Germany

5 Institute of Training Science and Sport Informatics, German Sport University Cologne, Cologne, Germany

Corresponding author:

Monoem Haddad

Department of Sport Science Program, Qatar University, College of Arts and Sciences

PO. Box 2713, Doha, Qatar

E-mail: mhaddad@qu.edu.qa

\section{Summary}

Background: The aim of the study was to determine whether a combination of strength training (ST) and local vibration (LV) improved the isometric maximum force of arm flexor muscles. ST was applied to the left arm of the subjects; LV was applied to the right arm of the same subjects. The main aim was to examine the effect of LV during a dumbbell biceps curl (Scott Curl) on isometric maximum force of the opposite muscle among the same subjects. It is hypothesized, that the intervention with LV produces a greater gain in isometric force of the arm flexors than ST.

Methods: Twenty-seven collegiate students participated in the study. The training load was $70 \%$ of the individual $1 \mathrm{RM}$. Four sets with 12 repetitions were performed three times per week during four weeks. The right arm of all subjects represented the vibration trained body side (VS) and the left arm served as the traditional trained body side (TTS).

Results: A significant increase of isometric maximum force in both body sides (Arms) occurred. VS, however, significantly increased isometric maximum force about $43 \%$ in contrast to $22 \%$ of the TTS. Conclusion: The combined intervention of ST and LC improves isometric maximum force of arm flexor muscles.

Level of evidence: III.

KEY WORDS: concentric elbow flexion, local body vibration, mechanical vibration, one repetition maximum.

\section{Introduction}

To optimise strength performance in trained and nontrained subjects, recent attention in strengh training (ST) has focused on adding mechanical vibration to the whole body (or parts of it) when being engaged in strength exercises ${ }^{1-3}$. Generally speaking, vibration is seen as a mechanical transmission of energy to the body. It stimulates and forces body muscles to contract and relax dozens of times each second ${ }^{4}$. Mechanical vibration exposure for muscles can elicit a tonic vibration reflex (TVR) which is a muscular reflex ${ }^{5}$. This acute effect might be considered as a part of the stretching reflex ${ }^{1}$ that involves the stimulation of the muscle spindle and the activation of neural signs and muscle fibres through large alpha motor neurons. This is supported by a significant dependency between EMG activity of thigh muscles (except musclus rectus femoris) and the acceleration loads of the applied vibrations during Whole-Body-Vibrations (WBV). An increase of acceleration load while standing on a vibration platform results in an increased of thigh muscle activity (except for musculus rectus femoris) ${ }^{6}$.

WBV training is an indirect transmission of vibratory stimulus through the body tissues via the feet. This alternative kind of training has been used in sport and rehabilitation ${ }^{7}$. Positive effects of WBV have been observed on muscular strength, power and jumping ability after long-term use, with greater improvements shown with untrained and elderly individuals ${ }^{1}$. Furthermore, positive to no effects of short-term WBV on strength of knee extensors and vertical jumping performance have been shown ${ }^{8-11}$. Houston et al. ${ }^{12}$ explained that WBV training may improve hamstring flexibility. Recently, Padulo et al. ${ }^{13}$ revealed a significant alteration of running kinematics of trained 
Marathon runners after 10 minutes of WBV training with $45 \mathrm{~Hz}$, resulting in a decrease of stride length of the participants in this study. Furthermore, selected parameters of repeated sprint ability, such as total sprint time, worst sprint time and fatigue index, improved significantly for junior soccer players after applying approximately 15 seconds of WBV during the recovery period while performing six maximal 40 meter shuttle sprints, which were separated by 20 seconds rest time ${ }^{14}$.

During WBV transmission, energy is absorbed by the soft tissues, thus reducing the vibration which might reduce the vibratory stimulus to the muscles distal to the vibration sources ${ }^{15}$. Hence, these muscles might be exposed to an insufficient vibration load to elicit performance enhancement. Local vibration (LV) which stimulates specific segments of the body might affect target muscles more intensively than WBV ${ }^{16}$. This type of vibration directly applied to target muscles or their antagonist might reduce dissipation of the vibration energy ${ }^{3}$. Therefore, target muscles can be stimulated more efficiently, resulting in the tonic vibration reflex (TVR) and, consequently, increasing the acute and chronic muscles' strength production. Many studies demonstrated significant chronic increases in the muscular force when conventional ST is combined with the use of mechanical vibration, and these increases can be acute or chronic ${ }^{1-3}$.

The responses to both types of vibration training (WBV and LV) may lead to increased motor unit recruitment, increased firing frequency and/or improved synchronisation, thereby permitting a quicker and more forceful muscle contraction when the muscle is rapidly stretched. Some Authors ${ }^{5}$ confirmed reflex answers during vibration; others, however proposed that relaxation of the antagonist muscle presents further reasons for greater force development of the agonist muscle ${ }^{17}$ thus resulting in an improvement of coordination between agonist and antagonist ${ }^{5}$. This can also be explained as a better synchronisation of the muscular activity ${ }^{18,19}$.

In the study of Silva et al. ${ }^{20}$, LV has been applied in the opposite direction of the resultant muscle contraction force vector. It has been shown that this use of vibration might be more efficient, since the addition of vibration on the resultant force vector of resistance might yield short eccentric effects in all the muscles that are liable to the specific movement, which might in turn increase the stimulation of the muscle spindles in target muscles.

In this context, the main aim of present study was to examine the influence of LV implemented by the antagonist muscle (i.e., triceps brachii muscle) on isometric maximum force of the agonist muscle (i.e., Biceps brachii) on the same subjects.

\section{Materials and methods}

\section{Subjects}

Thirty collegiate students belonging to the sport science department at the University took part in the study. The sample consisted of 19 male students (Mean \pm SD, Age $24.2 \pm 2.9$ y, Height $179.5 \pm 7.2 \mathrm{~cm}$, Body mass $75.6 \pm 8.7 \mathrm{~kg}$ ) and 11 female students (Mean \pm SD, Age $23.8 \pm 1.8$ y, Height $164.5 \pm 8.1 \mathrm{~cm}$, Body mass $60.1 \pm 7.9 \mathrm{~kg}$ ). All participants had good experience in ST. Prior to the study, all participants were informed about the potential risks and benefits associated with participation, and all signed a written informed consent form, agreeing with the protocol procedures and publication of the data. The study was conducted according to the ethical principles line of the Declaration of Helsinki and meets the ethical standards of this Journal, which were published by Padulo et al. ${ }^{21}$. The study protocol was approved by the Clinical Research Ethics Committee of the German Sport University Cologne before the commencement of the assessments. All subjects were fully accustomed to the procedures used in this research and were informed they could withdraw from the study in any time without penalty. Two male students and 1 female student made use of their right to withdraw from the study during the second week. Therefore, $N=27$ students (17 males, 10 females) completed the study, and their data were analyzed.

\section{Vibration technique}

In order to study whether the LV applied on the antagonist muscle during a dumbbell biceps curl (Scott Curl) has an effect on the isometric maximum force of the agonist muscle among the same subjects, the right arm was used as the experimental arm (VS, Vibration body-side) and the left arm was used as the control arm (TTS, Traditional Trained bod-side). Each test-person trained the right and the left arm. The right arm was exposed to local vibration stimulation through the triceps brachii (Fig. 1). The left arm of the same subject performed identical resistance training without vibration.

\section{Training protocol with vibration}

During training, the subjects' triceps brachii was placed on the vibrating surface. The elbow did not have contact with the vibration pad. In this position each subject performed 4 sets of Scott Curls exercise with 12 repetitions. The exercise was described as concentric elbow flexion: the positive work of the biceps brachii is a concentric contraction, whereas the triceps brachii contracts slightly eccentrically to control the movement ${ }^{22}$. The load is determined with $70 \%$ 1RM (Fig. 1, right picture). The movement speed of the exercise was self-determined by the participant ${ }^{23}$. The additional training weight was determined for both arms over the 1RM method ${ }^{24,25}$ by using a dumbbell. The 1RM for each subject was determined in three to five trials. A five minute recovery period was applied prior to each trial. All 1RM have been met within five trials.

The development of maximal force was tested during the $2^{\text {nd }}$ and $3^{\text {rd }}$ weeks in the training interval, at the end of the intervention phase and 2 weeks after the training ended. The intra-day effects of the testing 


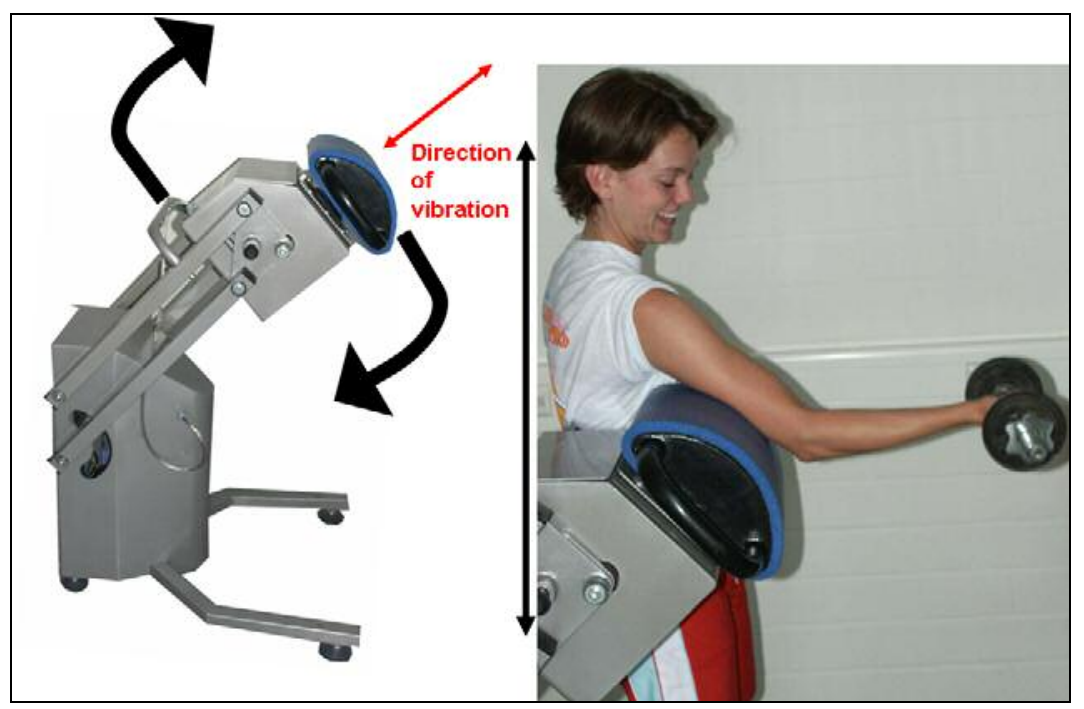

Figure 1. Left: Vibration generator Medic Swing (Mechatronic). Right: Training of the biceps.

and training were not controlled due to the different schedules of the participants, although recommended differently ${ }^{26}$. The frequency increased during every week with a constant amplitude of $4 \mathrm{~mm}$ peak-topeak displacement: $18 \mathrm{~Hz}, 24 \mathrm{~Hz}, 30 \mathrm{~Hz}$ and $36 \mathrm{~Hz}$ for $1^{\text {st }}, 2^{\text {nd }}, 3^{\text {rd }}$ and $4^{\text {th }}$ week, respectively. The acceleration for the frequency of $18 \mathrm{~Hz}$ equalled $2.6 \mathrm{~g}$. Three training sessions were scheduled per week. The training weight for the first training week was adjusted at $70 \%$ of the individually achieved $1 \mathrm{RM}$ value. During the first training week maximal force was measured once. Training weight was adjusted in relation to the test results of each week. A training load of $70 \%$ of the individual $1 \mathrm{RM}$ was maintained throughout the intervention period.

\section{Testing procedures}

During the $1^{\text {st }}$ week of the pretesting phase before training, each subject additionally performed three measurements of isometric force of the biceps brachii of both arms in order to exclude adaptations due to the familiarization of the testing protocol by the subjects. These test results are not part of $\mathrm{T} 1$, which describes the entry data for the intervention phase. The actual T1 was performed prior to the first training with a resting interval of 72 hours before the first session of training commenced. T2 and T3 measurements and the Posttest were performed after each training week with at least 72 hours rest after the last training session. After two weeks of detraining, the same tests were repeated during the Retest to measure changes in performance. The right arm of all subjects was tested before the left arm in the below described position with individually maximal effort. Isometric maximum force was measured three times for each arm for each test. The test with the highest isometric maximum force was considered for data analysis. Note that during this phase of testing, no vibrations were provided to the VS.
Maximal force of the biceps brachii was measured on a Scott Curl machine (Gym 80) which was equipped with a force sensor with a sampling rate of $100 \mathrm{~Hz}$ (Fa. Mechatronic). Figure 2 shows the position of the triceps brachii during the measurement on the Scott Curl machine. The angle between lower and upper arm was fixed at $170^{\circ}$. Reliability was measured in a classical test-retest design with 12 subjects during the pretests. The results of the test-retest scenario for isometric force revealed an intra-class correlation of ICC $=0.79(p<.01)$.

\section{Vibration equipment}

The vibrations were produced by the Medic Swing vibration generator (Mechatronic). This device works with a constant amplitude of $4 \mathrm{~mm}$, and allows a variable frequency between 18 and $38 \mathrm{~Hz}$ - according to manufacturer's information. This variable frequency has been applied starting with a relatively low frequency, in order to guard against overtraining due to an increased vibration load. The acceleration load ranged between $2.6 \mathrm{~g}(18 \mathrm{~Hz})$ and $10.4 \mathrm{~g}(36 \mathrm{~Hz})$. A high and low acceleration load intervention study shows modified muscle activity patterns as a response to changes in the excitation acceleration of input signals ${ }^{27}$. Its platform is convex and can be adjusted in height and angle. That is why it can be used for local vibrations of body segments and especially the upper body (Fig. 1).

\section{Data analysis}

A significance level of $a=5 \%$ was defined a priori for all results reported. The comparative performance metric for the current experiment was set to the variable of isometric force. In order to test the main hypothesis, an Analysis of Variance (ANOVA) with repeated measures was conducted. The factor Group/Body Side (vibration training vs traditional training) was treated as within-subject factor. Mea- 


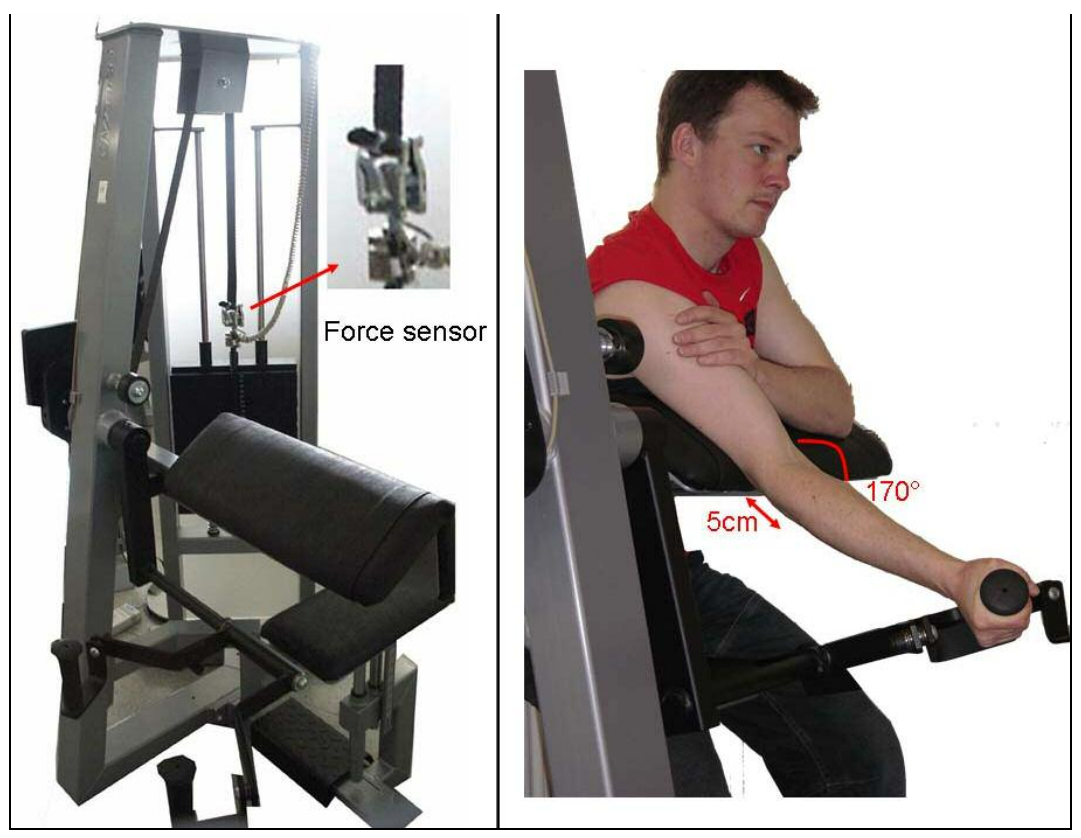

Figure 2. Test machine with force sensor (left); Right: measurement position.

surement (pretest vs T1 vs T2 vs T3 vs post-test vs. retest) was treated as within-subject factor. Cohen's $f$ was calculated as an effect size for all $F$-values larger than 1.0. Maximal force was used as the dependent variable. Post hoc analyses were carried out using the Tukey's HSD post-hoc test because of its greater power and control for Type I error inflation compared to other post-hoc tests.

\section{Results}

It was hypothesized that the intervention with LV would produce a greater gain in the isometric force of the arm flexors than traditional ST. In particular it was expected that VS would increase the isometric force faster (at an earlier measurement) than TTS. It was furthermore expected that, after 4 weeks of intervention, VS produces higher isometric force values than TTS and that the retention test reveals a minimized reduction of isometric force of the flexor muscles in VS.

Means and standard errors of the maximal isometric force values are presented in Figure 3.

The ANOVA revealed a significant main effect of Group/Body Side, $F(1,26)=22.48, p<.01$, Cohen's $f$ $=.92$, as well as a significant main effect of Measurement, $F(5,130)=25.87, p<.01$, Cohen's $f=.99$. The body side that was exposed to vibration training exhibited on average higher force production values (Mean \pm SE: $364.25 \pm 19.28 \mathrm{~N}$ ) than the body side that was not exposed to vibration training (330.21 \pm $19.36 \mathrm{~N}$ ) (see Table I). Post-hoc analysis for measurement revealed that force production values significantly increased from pretest to posttest. The value for retention test was significantly lower than the value for the posttest.
In addition, the ANOVA revealed a significant interaction effect of Group/Body Side x Measurement on force production, $F(5,130)=8.97, p<.01$, Cohen's $f$ $=.58$. Force production values were significantly higher for the body side that was exposed to vibration training in T2, in the post test, and in the retest (Tab. 1). Force production values were, however, neither significantly different between both body sides at the pretest, at T1, nor at T3.

\section{Discussion}

The main aim of the study was to test the effect of LV during a dumbbell biceps curl (Scott Curl) on the isometric maximum force of the opposite muscle among the same set of subjects. The primary result of the study showed that dynamic ST combined with LV presents an effective mechanism for the improvement of isometric maximum force. The VS (right arms) showed an increase of $43.2 \%(133 \mathrm{~N})$ of isometric strength between the first test T1 and the post-test T5 in contrast to $22 \%(68 \mathrm{~N})$ for the TTS. These gains differ significantly from each other in favor of VS. Several single cases within the VS show even higher improvement rates of isometric maximum force of up to $64 \%$. In general, the higher training intensity, which can be attributed to reflexes, is one explanation for the higher improvement of the vibration group. As the vibrations are generated near the trained muscle, this effect is increased. Another reason for the higher maximum force values of VS can be found in the relaxation of the antagonist. Since the vibrations were directly applied to the antagonistic m. triceps brachii, its inhibitory effect probably was minimized. Thus an improvement of coordination between agonist and antagonist is indicated 5 . Another advantage is the 


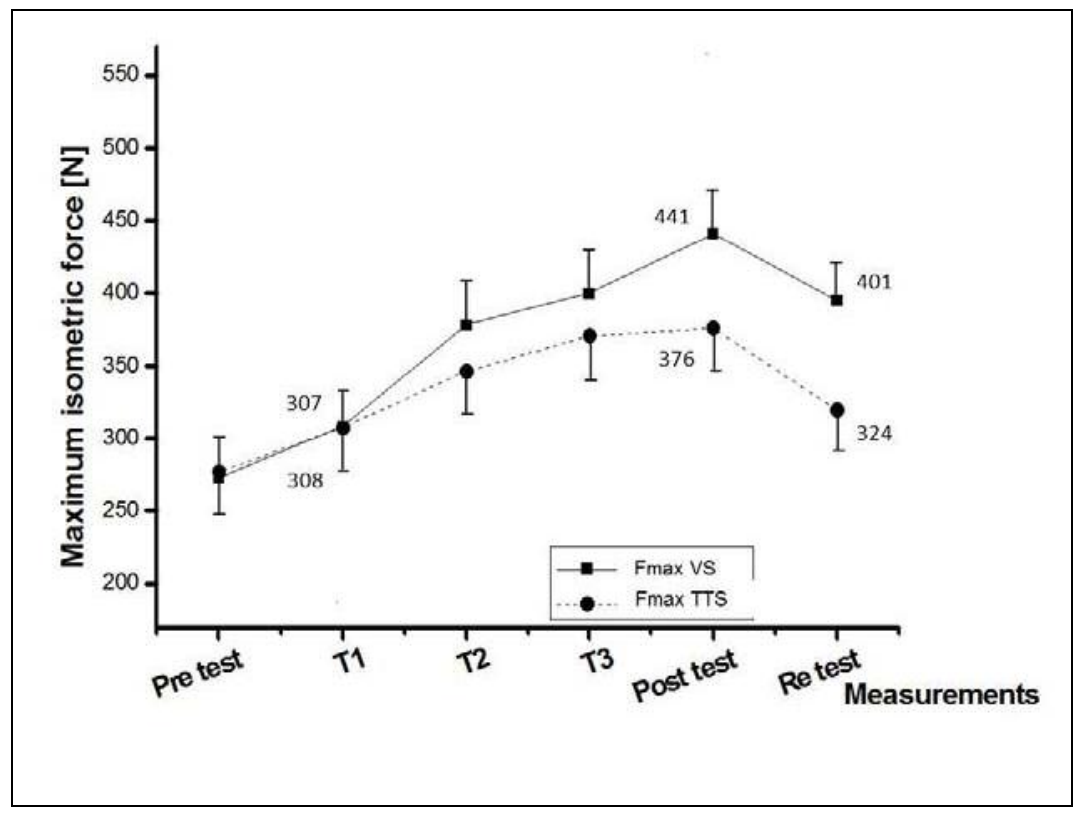

Figure 3. Means and standard error of the VS and TTS group.

Table I. Means and standard errors (SE) of maximum isometric force during pretest, entry test (T1), $2^{\text {nd }}$ and $3^{\text {rd }}$ week tests (T2, T3), posttest and retest ( 2 weeks after end of training) Note: VS = vibration trained body side, TTS traditional trained body side.

\begin{tabular}{lllllll}
\hline & Pretest & T1 & T2 & T3 & Posttest & Retest \\
\hline VS & 269.02 & 307.91 & 376.01 & 390.96 & 440.90 & 400.66 \\
Mean \pm SE & \pm 20.49 & \pm 19.58 & \pm 23.68 & \pm 23.31 & \pm 23.98 & \pm 20.93 \\
TTS & 270.79 & 308.12 & 338.01 & 364.44 & 375.93 & 323.93 \\
Mean \pm SE & \pm 23.48 & \pm 21.28 & \pm 22.03 & \pm 23.61 & \pm 21.75 & \pm 17.36 \\
\hline
\end{tabular}

better tolerability of local stimulation in contrast to whole body stimulation since local effects are easier to control. In some investigations in literature ${ }^{3,28}$ no effect or even a remarkable decrease of maximal force and strength related abilities is measured in the final test. Drummond et al. ${ }^{3}$ have shown that the application of local vibration does not change the chronic effects of dynamic ST in untrained individuals after 12 weeks. The main reason for this result often can be found in a too long and/or too intensive training schedule. In contrast to these previous findings, the current study shows an increase of maximum force in the final test and a decrease of approx. 14 to $18 \%$ in both groups in the re-test after 2 weeks without training. This indicates that the training parameters were well chosen, i.e., not too intensive. The re-test also revealed that there is still a remarkable increase of isometric maximum strength left in VS $(28.2 \%$ in retest compared to $T$ 1). In conclusion, dynamic ST combined with LV might improve the isometric maximum strength of the opposite muscle. Since the results of this study with LV are promising, further investigations of more parameters such as speed strength, speed, and reactivity might is warranted.

\section{Acknowledgements}

The Authors want to express their thanks to all the students of the German Sport University Cologne, who participated in this study. No external financial support has been received.

\section{Conflicts of interest}

The Authors declare no conflicts of interest concerning this article.

\section{References}

1. Marin PJ, Rhea MR. Effects of vibration training on muscle power: a meta-analysis. Journal of strength and conditioning research/National Strength \& Conditioning Association. 2010:871-878.

2. Rittweger J, Beller G, Felsenberg D. Acute physiological effects of exhaustive whole-body vibration exercise in man. Clinical physiology. 2000;2:134-142.

3. Drummond MD, Couto BP, Augusto IG, Rodrigues SA, Szmuchrowski LA. Effects of 12 weeks of dynamic strength train- 
ing with local vibration. Eur J Sport Sci. 2014;7:695-702.

4. Mester J, Spitzenpfeil P, Yue Z. Vibration Loads: Potential for Strength and Power Development. In: Komi P, editor. Strength and Power in Sport, Second Edition. Oxford: International Olympic Committee. 2008;488-501.

5. Martin BJ, Park HS. Analysis of the tonic vibration reflex: influence of vibration variables on motor unit synchronization and fatigue. European journal of applied physiology and occupational physiology. 1997;6:504-511.

6. Di Giminiani R, Masedu F, Padulo J, Tihanyi J, Valenti M. The EMG activity-acceleration relationship to quantify the optimal vibration load when applying synchronous whole-body vibration. Journal Of Electromyography \& Kinesiology. 2015;6:853859.

7. Rehn B, Lidstrom J, Skoglund J, Lindstrom B. Effects on leg muscular performance from whole-body vibration exercise: a systematic review. Scandinavian journal of medicine \& science in sports. 2007;1:2-11.

8. Petit PD, Pensini M, Tessaro J, Desnuelle C, Legros P, Colson SS. Optimal whole-body vibration settings for muscle strength and power enhancement in human knee extensors. Journal of electromyography and kinesiology: official journal of the International Society of Electrophysiological Kinesiology. 2010; 6:1186-1195.

9. Da Silva-Grigoletto ME, Vaamonde DM, Castillo E, Poblador MS, Garcia-Manso JM, Lancho JL. Acute and cumulative effects of different times of recovery from whole body vibration exposure on muscle performance. Journal of strength and conditioning research / National Strength \& Conditioning Association. 2009;7:2073-2082.

10. Savelberg HH, Keizer HA, Meijer K. Whole-body vibration induced adaptation in knee extensors; consequences of initial strength, vibration frequency, and joint angle. Journal of strength and conditioning research/National Strength \& Conditioning Association. 2007;2:589-93.

11. Delecluse C, Roelants M, Diels R, Koninckx E, Verschueren S. Effects of whole body vibration training on muscle strength and sprint performance in sprint-trained athletes. International journal of sports medicine. 2005;8:662-668.

12. Houston MN, Hodson VE, Adams KK, Hoch JM. The effectiveness of whole-body-vibration training in improving hamstring flexibility in physically active adults. J Sport Rehabil. 2015;1:77-82.

13. Padulo J, Filingeri $D$, Chamari $K$, et al. Acute effects of wholebody vibration on running gait in marathon runners. Journal Of Sports Sciences. 2014;12:1120-1126.

14. Padulo J, Di Giminiani R, lbba G, et al. The Acute Effect of Whole Body Vibration on Repeated Shuttle-Running in Young Soccer Players. International Journal Of Sports Medicine.
2014;1:49-54.

15. Pollock RD, Woledge RC, Mills KR, Martin FC, Newham DJ. Muscle activity and acceleration during whole body vibration: effect of frequency and amplitude. Clin Biomech (Bristol, Avon) 2010;25:840-846.

16. Peer KS, Barkley JE, Knapp DM. The acute effects of local vibration therapy on ankle sprain and hamstring strain injuries. The Physician and sportsmedicine. 2009;4:31-38.

17. Eklund G, Hagbarth KE. Normal variability of tonic vibration reflexes in man. Exp Neurol. 1966; 1:80-92. PubMed PMID: 5923486.

18. Lippold OC, Redfearn JW, Vuco J. The rhythmical activity of groups of motor units in the voluntary contraction of muscle. $J$ Physiol. 1957;3:473-487.

19. Bosco $C$, Colli $R$, Introini $E$, et al. Adaptive responses of human skeletal muscle to vibration exposure. Clinical physiology. 1999;2:183-187.

17. Silva HR, Couto BP, Szmuchrowski LA. Effects of mechanical vibration applied in the opposite direction of muscle shortening on maximal isometric strength. Journal of strength and conditioning research/National Strength \& Conditioning Association. 20084:1031-1036.

21. Padulo J, Oliva F, Frizziero A, Maffulli N. Muscles, Ligaments and Tendons Journal - Basic principles and recommendations in clinical and field Science Research: 2016 Update. MLTJ. 2016;6(1):1.

22. Padulo J, Laffaye G, Chamari K, Concu A. Concentric and eccentric: muscle contraction or exercise. Sports Health. 2013; 4:306.

23. Padulo J, Mignogna P, Mignardi S, Tonni F, D'Ottavio S. Effect of different pushing speeds on bench press. International journal of sports medicine, 2012;5:376-380.

24. Padulo J, Laffaye G, Chaouachi A, Chamari K. Bench Press exercise: the key points. J Sports Med Phys Fitness. 2014:6: 604-608.

25. Baechle TR, Earle RW, National S, Conditioning A. Essentials of strength training and conditioning. Leeds: Human Kinetics. 2008.

26. Ammar A, Chtourou H, Trabelsi K, et al. Temporal specificity of training: intra-day effects on biochemical responses and Olympic-Weightlifting performances. Journal of sports sciences. 2015;4:358-368.

27. Padulo J, Di Giminiani R, Dello lacono A, et al. Lower Arm Muscle Activation During Indirect-Localized Vibration: The Influence Of Skill Levels When Applying Different Acceleration Loads. Frontiers in Physiology. 2016;7:242.

28. Yue Z, Mester J. A model analysis of internal loads, energetics, and effects of wobbling mass during the whole-body vibration. J Biomech. 2002:639-647. 